\title{
PROVERBS RELATED TO MONEY IN THREE SLAVIC LINGUOCULTURES: BULGARIAN, RUSSIAN, AND SLOVAK
}

\section{IVO PANCHEV}

INSTITUTE FOR BUlgarian LANGUAGE, BULgaRIAN ACADEMY OF SCIENCES

ivopanchev@ibl.bas.bg

This article proposes an analysis of a number of Bulgarian, Russian, and Slovak proverbs and sayings with lexemes denoting the concept of money. Money is a phenomenon worthy of research both in a linguistic and in a cultural perspective. Using a corpus of proverbs and sayings, the author has been able to construct Bulgarian language and culture-based culturemes so that they could be compared with Russian and Slovak counterparts constructing the same culturemes. The analysis reveals a number of differences in the comparisons and metaphors used, or, to put it in more general terms - in the representation of proverbs and sayings that otherwise correspond to each other at the culturemic level.

Keywords: Bulgarian, Russian, and Slovak proverbs, culturemes, linguocultural studies

\section{REFERENCES}

Dankov 1978: Dankov, B. Balgarskite moneti. - Rabotnichesko delo, № 96, s. 5.

Kirilova 2017: Kirilova, Y. Predstavata za uma v balgarskata ezikova kartina na sveta. Sofia, Diomira.

Konstantinova 2011: Konstantinova, D. Ironiyata kato nachin za vazpriemane na sveta: varhu material ot balgarskata i slovashkata frazeologiya. V. Tarnovo, UI "Sv. sv. Kiril i Metodiy".

Konstantinova 2008: Konstantinova, D. Za nyakoi ironichni frazeologizmi v balgarskia i slovashkia ezik. - In: Izsledvania po frazeologiya, leksikologiya i leksikografiya. V pamet na prof. dfn Keti Ankova-Nicheva. Sofia, Akad. Izdatelstvo „Prof. M. Drinov“.

Panchev 2018: Panchev, I. Sledite na kontsepta pari v dve slavyanski lingvokulturi: balgarskata i ruskata. - LIMES SLAVICUS 3. Kulturni kontsepti na slavyanstvoto. Shumen: UI "Episkop Konstantin Preslavski”, s. 37-46.

Petrova 2013: Petrova, $R$. Balgarski paremii za prismeh i pohvala. Lingvokulturno izsledvane (skala na kulturemite). Ruse, Izdatelski tsentar pri RU "Angel Kanchev".

Petrova 2006: Petrova, R. Kulturemata - edinitsa za lingvokulturologichni sapostavitelni izsledvaniya (varhu material ot angliyski i balgarski poslovitsi). $<\mathrm{http} / /$ lingvocult.uni-ruse.bg/pubs/ZA\%20KULTUREMATA-R.Petrova.pdf> 
Stoev 1967: Stoev, G. Tsenata na zlatoto. Sofia, Balgarski pisatel.

Vlahov 1977: Vlahov, S. Ruski poslovitsi s balgarski prevodi i saotvetki. Sofia, Nauka i izkustvo.

$\triangle$ Assist. Prof. Ivo Panchev, PhD Department of General and Comparative Studies Institute for Bulgarian Language, Bulgarian Academy of Sciences 52 Shipchenski prohod, B1. 17, Sofia 1113, Bulgaria 